BA-08-05

KEK-TH-1251

\title{
Higgs Boson Mass Bounds in the Standard Model with Type III and Type I Seesaw
}

\author{
Ilia Gogoladze ${ }^{a}$, Nobuchika Okada ${ }^{b}$ and Qaisar Shafi ${ }^{a}$ \\ ${ }^{a}$ Bartol Research Institute, Department of Physics and Astronomy, \\ University of Delaware, Newark, DE 19716, USA \\ ${ }^{b}$ Theory Division, KEK, Tsukuba 305-0801, Japan
}

\begin{abstract}
In type III seesaw utilized to explain the observed solar and atmospheric neutrino oscillations the Standard Model (SM) particle spectrum is extended by introducing three $\mathrm{SU}(2)_{L}$ triplet fermion fields. This can have important implications for the SM Higgs boson mass $\left(M_{H}\right)$ bounds based on vacuum stability and perturbativity arguments. We compute the appropriate renormalization group equations for type III seesaw, and then proceed to identify regions of the parameter space such that the SM Higgs boson mass window is enlarged to $125 \mathrm{GeV}$ $\lesssim M_{H} \lesssim 174 \mathrm{GeV}$, with the type III seesaw scale close to TeV. We also display regions of the parameter space for which the vacuum stability and perturbativity bounds merge together for large neutrino Yukawa couplings. Comparison with type I seesaw is also presented.
\end{abstract}


In a recent paper [1] we studied the impact of type II seesaw [2] on the predictions for the SM Higgs boson mass based on vacuum stability and perturbativity arguments [3]. It was shown in [1] that a Higgs boson mass as low as $114.4 \mathrm{GeV}$ (LEP2 bound [4]) can be realized in type II seesaw. This is to be contrasted with a lower bound of $131 \mathrm{GeV}$ realized both in the absence of any seesaw or with type I seesaw [5]. Thus, it would seem that discovery of a relatively 'light' Higgs boson (with mass well below $131 \mathrm{GeV}$ ) would signal the presence of new physics which may well be related to the presence of type II seesaw around the TeV scale or higher [1]. Since type II seesaw is based on $\mathrm{SU}(2)_{L}$ triplet scalar fields it is conceivable that some of these scalars may be found at the LHC.

In this paper we wish to continue our investigation of the SM Higgs boson mass (in the light of neutrino oscillations [6]) by focusing our attention on type III seesaw [7]. This mechanism is very similar to type I seesaw except that the SM singlet fermion fields (right handed neutrinos) are replaced by $\mathrm{SU}(2)_{L}$ triplet fermions carrying zero hypercharge.

The appearance of new fermion fields in type III seesaw modifies the evolution of the various SM couplings. In particular, the renormalization group equation (RGEs) of the Higgs quartic coupling and the $\mathrm{SU}(2)_{L}$ gauge coupling will be altered, and this will play an important role in modifying the vacuum stability $(\gtrsim 131 \mathrm{GeV})$ and perturbativity $(\lesssim 171 \mathrm{GeV})$ bounds obtained by ignoring neutrino oscillations.

The implication of type I seesaw for the Higgs boson mass has been investigated in Ref. [8] employing one-loop RGEs. It was shown that the Higgs boson mass from the vacuum stability bound is pushed up as the Dirac Yukawa coupling becomes larger, and eventually merges with the perturbativity bound around $M_{H} \simeq 170 \mathrm{GeV}$. In our previous work [1], we have investigated the Higgs boson mass bounds in type II seesaw. The triplet scalar $\Delta$ in type II seesaw has both cubic and quartic couplings with the Higgs doublet and we have studied the coupled RGEs involving the Higgs doublet and $\Delta$. The cubic and quartic couplings can drastically change the RGE evolution of the Higgs quartic coupling and in particular, the perturbativity bound is pushed down as the couplings become larger and eventually merges with the vacuum stability bounds. Furthermore, for a plausible choice of parameters, the resultant Higgs boson mass can be as low as the LEP2 bound of $114.4 \mathrm{GeV}$ [4].

The basic structure of type III seesaw is similar to type I seesaw, except that instead of the singlet right-handed neutrinos, $\mathrm{SU}(2)_{L}$ triplet fermions with zero hypercharge are introduced. Thus, we may expect similar results for the Higgs boson mass bounds as in type I seesaw. This turns out to be true for large Yukawa couplings. However, as we will show below, the range of the Higgs boson mass from vacuum stability and perturbativity bounds is enlarged from the SM one for small Yukawa couplings. This is in contrast with type I seesaw in which the Higgs 
boson mass bounds reduce to the SM ones for small Yukawa couplings.

We begin by introducing three generations of fermions $\psi_{i}(i=1,2,3)$, which transforms as $(\mathbf{3}, 0)$ under the electroweak gauge group $\mathrm{SU}(2)_{L} \times \mathrm{U}(1)_{Y}$ :

$$
\psi_{i}=\sum_{a} \frac{\sigma^{a}}{2} \psi_{i}^{a}=\frac{1}{2}\left(\begin{array}{cc}
\psi_{i}^{0} & \sqrt{2} \psi_{i}^{+} \\
\sqrt{2} \psi_{i}^{-} & -\psi_{i}^{0}
\end{array}\right) .
$$

For simplicity, we assume in this paper that the three triplet fermions are degenerate in mass $(M)$ so that their mass matrix is proportional to the 3 by 3 unit matrix, $\mathbf{M}=M \times \mathbf{1}_{3 \times 3}$.

With canonically normalized kinetic terms for the triplet fermions, we introduce the Yukawa coupling

$$
\mathcal{L}_{Y}=y_{i j} \overline{\ell_{i}} \psi_{j} \Phi
$$

where $\Phi$ is the Higgs doublet. At low energies, the heavy triplet fermions are integrated out and the effective dimension five operator is generated by the seesaw mechanism. After electroweak symmetry breaking, the light neutrino mass matrix is obtained as

$$
\mathbf{M}_{\nu}=-\frac{v^{2}}{8} \mathbf{Y}_{\nu}^{T} \mathbf{M}^{-1} \mathbf{Y}_{\nu}=-\frac{v^{2}}{8 M} \mathbf{Y}_{\nu}^{T} \mathbf{Y}_{\nu}
$$

where $v=246.2 \mathrm{GeV}$ is the VEV of the Higgs doublet, $\mathbf{Y}_{\nu}=y_{i j}$ is a $3 \times 3$ Yukawa matrix, and we have used the assumption $\mathbf{M}=M \times \mathbf{1}_{3 \times 3}$ in the last equality.

At energies higher than the triplet fermion mass, the SM RGEs should be modified to include contributions from the triplet fermions, so that the RGE evolution of the Higgs quartic coupling is altered. We have computed the one-loop RGEs for the new contributions associated with type III seesaw scenario. In our analysis, we employ two-loop RGEs for the SM couplings.

For a renormalization scale $\mu<M$, the triplet fermions are decoupled. For the three SM gauge couplings, we have

$$
\frac{d g_{i}}{d \ln \mu}=\frac{b_{i}}{16 \pi^{2}} g_{i}^{3}+\frac{g_{i}^{3}}{\left(16 \pi^{2}\right)^{2}}\left(\sum_{j=1}^{3} B_{i j} g_{j}^{2}-C_{i} y_{t}^{2}\right),
$$

where $g_{i}(i=1,2,3)$ are the SM gauge couplings,

$$
b_{i}=\left(\frac{41}{10},-\frac{19}{6},-7\right), \quad B_{i j}=\left(\begin{array}{ccc}
\frac{199}{50} & \frac{27}{10} & \frac{44}{5} \\
\frac{9}{10} & \frac{35}{6} & 12 \\
\frac{11}{10} & \frac{9}{2} & -26
\end{array}\right), \quad C_{i}=\left(\frac{17}{10}, \frac{3}{2}, 2\right),
$$

and we have included the contribution from the top Yukawa coupling $\left(y_{t}\right)$. The top quark pole mass is taken to be the central value $M_{t}=172.6 \mathrm{GeV}$, 9], with $\left(\alpha_{1}, \alpha_{2}, \alpha_{3}\right)=(0.01681,0.03354,0.1176)$ at the Z-pole $\left(M_{Z}\right)$ [10]. For the top Yukawa coupling, we have [11,

$$
\frac{d y_{t}}{d \ln \mu}=y_{t}\left(\frac{1}{16 \pi^{2}} \beta_{t}^{(1)}+\frac{1}{\left(16 \pi^{2}\right)^{2}} \beta_{t}^{(2)}\right) \text {. }
$$


Here the one-loop contribution is

$$
\beta_{t}^{(1)}=\frac{9}{2} y_{t}^{2}-\left(\frac{17}{20} g_{1}^{2}+\frac{9}{4} g_{2}^{2}+8 g_{3}^{2}\right)
$$

while the two-loop contribution is given by

$$
\begin{aligned}
\beta_{t}^{(2)}= & -12 y_{t}^{4}+\left(\frac{393}{80} g_{1}^{2}+\frac{225}{16} g_{2}^{2}+36 g_{3}^{2}\right) y_{t}^{2} \\
& +\frac{1187}{600} g_{1}^{4}-\frac{9}{20} g_{1}^{2} g_{2}^{2}+\frac{19}{15} g_{1}^{2} g_{3}^{2}-\frac{23}{4} g_{2}^{4}+9 g_{2}^{2} g_{3}^{2}-108 g_{3}^{4} \\
& +\frac{3}{2} \lambda^{2}-6 \lambda y_{t}^{2} .
\end{aligned}
$$

In solving Eq. (6) , the initial top Yukawa coupling at $\mu=M_{t}$ is determined from the relation between the pole mass and the running Yukawa coupling [12], [13],

$$
M_{t} \simeq m_{t}\left(M_{t}\right)\left(1+\frac{4}{3} \frac{\alpha_{3}\left(M_{t}\right)}{\pi}+11\left(\frac{\alpha_{3}\left(M_{t}\right)}{\pi}\right)^{2}-\left(\frac{m_{t}\left(M_{t}\right)}{2 \pi v}\right)^{2}\right)
$$

with $y_{t}\left(M_{t}\right)=\sqrt{2} m_{t}\left(M_{t}\right) / v$, where $v=246.2 \mathrm{GeV}$. Here, the second and third terms in parenthesis correspond to one- and two-loop QCD corrections, respectively, while the fourth term comes from the electroweak corrections at one-loop level. The numerical values of the third and fourth terms are comparable (their signs are opposite). The electroweak corrections at two-loop level and the three-loop QCD corrections [13, are of comparable and sufficiently small magnitude [13] to be safely ignored.

The RGE for the Higgs quartic coupling is given by [11,

$$
\frac{d \lambda}{d \ln \mu}=\frac{1}{16 \pi^{2}} \beta_{\lambda}^{(1)}+\frac{1}{\left(16 \pi^{2}\right)^{2}} \beta_{\lambda}^{(2)}
$$

with

$$
\beta_{\lambda}^{(1)}=12 \lambda^{2}-\left(\frac{9}{5} g_{1}^{2}+9 g_{2}^{2}\right) \lambda+\frac{9}{4}\left(\frac{3}{25} g_{1}^{4}+\frac{2}{5} g_{1}^{2} g_{2}^{2}+g_{2}^{4}\right)+12 y_{t}^{2} \lambda-12 y_{t}^{4}
$$

and

$$
\begin{aligned}
\beta_{\lambda}^{(2)}= & -78 \lambda^{3}+18\left(\frac{3}{5} g_{1}^{2}+3 g_{2}^{2}\right) \lambda^{2}-\left(\frac{73}{8} g_{2}^{4}-\frac{117}{20} g_{1}^{2} g_{2}^{2}+\frac{2661}{100} g_{1}^{4}\right) \lambda-3 \lambda y_{t}^{4} \\
& +\frac{305}{8} g_{2}^{6}-\frac{289}{40} g_{1}^{2} g_{2}^{4}-\frac{1677}{200} g_{1}^{4} g_{2}^{2}-\frac{3411}{1000} g_{1}^{6}-64 g_{3}^{2} y_{t}^{4}-\frac{16}{5} g_{1}^{2} y_{t}^{4}-\frac{9}{2} g_{2}^{4} y_{t}^{2} \\
& +10 \lambda\left(\frac{17}{20} g_{1}^{2}+\frac{9}{4} g_{2}^{2}+8 g_{3}^{2}\right) y_{t}^{2}-\frac{3}{5} g_{1}^{2}\left(\frac{57}{10} g_{1}^{2}-21 g_{2}^{2}\right) y_{t}^{2}-72 \lambda^{2} y_{t}^{2}+60 y_{t}^{6}
\end{aligned}
$$


The Higgs boson pole mass $M_{H}$ is determined by its relation to the running Higgs quartic coupling through the one-loop matching condition [14],

$$
\lambda\left(M_{H}\right) v^{2}=M_{H}^{2}\left(1+\Delta_{h}\left(M_{H}\right)\right),
$$

where

$$
\Delta_{h}\left(M_{H}\right)=\frac{G_{F}}{\sqrt{2}} \frac{M_{Z}^{2}}{16 \pi^{2}}\left[\frac{M_{H}^{2}}{M_{Z}^{2}} f_{1}\left(\frac{M_{H}^{2}}{M_{Z}^{2}}\right)+f_{0}\left(\frac{M_{H}^{2}}{M_{Z}^{2}}\right)+\frac{M_{Z}^{2}}{M_{H}^{2}} f_{-1}\left(\frac{M_{H}^{2}}{M_{Z}^{2}}\right)\right]
$$

The functions are given by

$$
\begin{aligned}
f_{1}(\xi)= & \frac{3}{2} \ln \xi-\frac{1}{2} Z\left(\frac{1}{\xi}\right)-Z\left(\frac{c_{w}^{2}}{\xi}\right)-\ln c_{w}^{2}+\frac{9}{2}\left(\frac{25}{9}-\frac{\pi}{\sqrt{3}}\right) \\
f_{0}(\xi)= & -6 \ln \frac{M_{H}^{2}}{M_{Z}^{2}}\left[1+2 c_{w}^{2}-2 \frac{M_{t}^{2}}{M_{Z}^{2}}\right]+\frac{3 c_{w}^{2} \xi}{\xi-c_{w}^{2}} \ln \frac{\xi}{c_{w}^{2}}+2 Z\left(\frac{1}{\xi}\right) \\
& +4 c_{w}^{2} Z\left(\frac{c_{w}^{2}}{\xi}\right)+\left(\frac{3 c_{w}^{2}}{s_{w}^{2}}+12 c_{w}^{2}\right) \ln c_{w}^{2}-\frac{15}{2}\left(1+2 c_{w}^{2}\right) \\
& -3 \frac{M_{t}^{2}}{M_{Z}^{2}}\left[2 Z\left(\frac{M_{t}^{2}}{M_{Z}^{2} \xi}\right)+4 \ln \frac{M_{t}^{2}}{M_{Z}^{2}}-5\right], \\
f_{-1}(\xi)= & 6 \ln \frac{M_{H}^{2}}{M_{Z}^{2}}\left[1+2 c_{w}^{4}-4 \frac{M_{t}^{4}}{M_{Z}^{4}}\right]-6 Z\left(\frac{1}{\xi}\right)-12 c_{w}^{4} Z\left(\frac{c_{w}^{2}}{\xi}\right)-12 c_{w}^{4} \ln c_{w}^{2} \\
& +8\left(1+2 c_{w}^{4}\right)+24 \frac{M_{t}^{4}}{M_{Z}^{4}}\left[\ln \frac{M_{t}^{2}}{M_{Z}^{2}}-2+Z\left(\frac{M_{t}^{2}}{M_{Z}^{2} \xi}\right)\right],
\end{aligned}
$$

with $s_{w}^{2}=\sin ^{2} \theta_{W}, c_{w}^{2}=\cos ^{2} \theta_{W}\left(\theta_{W}\right.$ denotes the weak mixing angle $)$ and

$$
Z(z)=\left\{\begin{array}{cc}
2 A \arctan (1 / A) & (z>1 / 4) \\
A \ln [(1+A) /(1-A)] & (z<1 / 4)
\end{array}\right.
$$

with $A=\sqrt{|1-4 z|}$.

For $\mu \geq M$, as previously mentioned, we include the one-loop contributions from the new fermion triplets. We have computed these new contributions for the wave function renormalization factors and vertex corrections for the neutrino Yukawa couplings, and for the Higgs quartic coupling, by employing dimensional regularization and $R_{\xi}$ gauge for the gauge boson propagators. The wave function renormalization factors of the SM Higgs doublet and lepton doublets receive new contributions from the neutrino Yukawa couplings:

$$
\delta Z_{\Phi}^{\text {new }}=-\frac{1}{16 \pi^{2} \epsilon}\left(\frac{3}{2} \operatorname{tr}\left[\mathbf{Y}_{\nu}^{\dagger} \mathbf{Y}_{\nu}\right]\right), \quad \delta Z_{\ell}^{\text {new }}=-\frac{1}{16 \pi^{2} \epsilon}\left(\frac{3}{4} \mathbf{Y}_{\nu} \mathbf{Y}_{\nu}^{\dagger}\right)
$$

The wave function renormalization factor for the triplet fermions is calculated to be

$$
\delta Z_{\psi}=-\frac{1}{16 \pi^{2} \epsilon}\left(\frac{1}{2} \mathbf{Y}_{\nu}^{\dagger} \mathbf{Y}_{\nu}+4 g_{2}^{2} \xi_{2}\right)
$$


where $\xi_{2}$ is a gauge parameter for $\mathrm{SU}(2)_{L}$. For the neutrino Yukawa coupling matrix we find

$$
\delta \mathbf{Y}_{\nu}=-\frac{1}{16 \pi^{2} \epsilon}\left[\frac{3}{10} g_{1}^{2} \xi_{1}+g_{2}^{2}\left(6+\frac{7}{2} \xi_{2}\right)\right]
$$

The Higgs quartic coupling receives a new contribution given by

$$
\delta \lambda=-\frac{1}{16 \pi^{2} \epsilon}\left(\frac{5}{4} \operatorname{tr}\left[\mathbf{Y}_{\nu}^{\dagger} \mathbf{Y}_{\nu}\right]\right)
$$

Consequently, we replace $b_{i}$ in Eq. (5) with

$$
b_{i}=\left(\frac{41}{10}, \frac{5}{6},-7\right) .
$$

In the RGE for the top Yukawa coupling, $\beta_{t}^{(1)}$ is modified, namely

$$
\beta_{t}^{(1)} \rightarrow \beta_{t}^{(1)}+\frac{3}{4} \operatorname{tr}\left[\mathbf{S}_{\nu}\right]
$$

through the new contribution to the wave function renormalization for the Higgs doublet. Here, $\mathbf{S}_{\nu}=\mathbf{Y}_{\nu}^{\dagger} \mathbf{Y}_{\nu}$ and its corresponding RGE is found to be

$$
16 \pi^{2} \frac{d \mathbf{S}_{\nu}}{d \ln \mu}=\mathbf{S}_{\nu}\left[6 y_{t}^{2}+\frac{3}{2} \operatorname{tr}\left[\mathbf{S}_{\nu}\right]-\left(\frac{9}{10} g_{1}^{2}+\frac{33}{2} g_{2}^{2}\right)+\frac{5}{4} \mathbf{S}_{\nu}\right] .
$$

The RGE of the Higgs quartic coupling acquires a new entry in Eq.(11),

$$
\beta_{\lambda}^{(1)} \rightarrow \beta_{\lambda}^{(1)}+3 \operatorname{tr}\left[\mathbf{S}_{\nu}\right] \lambda-\frac{5}{4} \operatorname{tr}\left[\mathbf{S}_{\nu}^{2}\right] .
$$

We next analyze the RGEs numerically and show how the vacuum stability and perturbativity bounds on Higgs boson mass are altered in the presence of type III seesaw. Fixing the cutoff scale as $M_{P l}=1.2 \times 10^{19} \mathrm{GeV}$, we define the vacuum stability bound as the lowest Higgs boson mass obtained from the running of the Higgs quartic coupling which satisfies the condition $\lambda(\mu) \geq 0$ for any scale between $M_{H} \leq \mu \leq M_{P l}$. On the other hand, the perturbativity bound is defined as the highest Higgs boson mass obtained from the running of the Higgs quartic coupling with the condition $\lambda(\mu) \leq \sqrt{4 \pi}$ for any scale between $M_{H} \leq \mu \leq M_{P l}$.

In order to see the effects of the neutrino Yukawa coupling on the Higgs boson mass bounds, let us first examine a toy model with $\mathbf{Y}_{\nu}=\operatorname{diag}\left(0,0, Y_{\nu}\right)$. In Fig. 1, the running Higgs mass, defined as $m_{H}(\mu)=\sqrt{\lambda(\mu)} v$ for the vacuum stability bound is depicted for various $Y_{\nu}$ values and a fixed seesaw scale $M=10^{13} \mathrm{GeV}$. Fig. 2 shows a running Higgs mass for the perturbativity bound for various $Y_{\nu}$ values and a fixed seesaw scale $M=10^{13} \mathrm{GeV}$. We find that as $Y_{\nu}$ is increased, the running Higgs mass for the vacuum stability bound shows a peak which 
reaches higher, while the change in the RGE evolution for the perturbativity bound is mild. This means that as $Y_{\nu}$ is increased, the vacuum stability and perturbativity bounds eventually merge. In other words, the window for the Higgs boson mass between the vacuum stability and perturbative bounds becomes narrower and is eventually closed as $Y_{\nu}$ becomes sufficiently large. This behavior is shown in Fig. 3.

Note that in Fig. 3, the precise values of the Higgs boson mass bounds are different from the $\mathrm{SM}$ ones, even in the limit $Y_{\nu} \rightarrow 0$. This is because the RGE of the $\mathrm{SU}(2)_{L}$ gauge coupling for $\mu \geq M$ is different from the SM one in the presence of the electroweak triplet fermions. We will see later that this effect becomes sizable as $M$ is lowered.

It is certainly interesting to consider more realistic cases so as to reproduce the current neutrino oscillation data. The light neutrino mass matrix is diagonalized by a mixing matrix $U_{M N S}$ such that

$$
\mathbf{M}_{\nu}=\frac{v^{2}}{8 M} \mathbf{S}_{\nu}=U_{M N S} D_{\nu} U_{M N S}^{T}
$$

with $D_{\nu}=\operatorname{diag}\left(m_{1}, m_{2}, m_{3}\right)$, where we have assumed, for simplicity, that the Yukawa matrix $\mathbf{Y}_{\nu}$ is real. We further assume that the mixing matrix has the so-called tri-bimaximal form [15],

$$
U_{M N S}=\left(\begin{array}{ccc}
\sqrt{\frac{2}{3}} & \sqrt{\frac{1}{3}} & 0 \\
-\sqrt{\frac{1}{6}} & \sqrt{\frac{1}{3}} & \sqrt{\frac{1}{2}} \\
-\sqrt{\frac{1}{6}} & \sqrt{\frac{1}{3}} & -\sqrt{\frac{1}{2}}
\end{array}\right)
$$

which is in very good agreement with the current best fit values of the neutrino oscillation data [6]. Let us consider two examples for the light neutrino mass spectrum, the hierarchical case and the inverted-hierarchical case. In the hierarchical case, we have

$$
D_{\nu} \simeq \operatorname{diag}\left(0, \sqrt{\Delta m_{12}^{2}}, \sqrt{\Delta m_{23}^{2}}\right),
$$

while for the inverted-hierarchical case, we choose

$$
D_{\nu} \simeq \operatorname{diag}\left(\sqrt{-\Delta m_{12}^{2}+\Delta m_{23}^{2}}, \sqrt{\Delta m_{23}^{2}}, 0\right)
$$

We fix the input values for the solar and atmospheric neutrino oscillation data as [6]

$$
\begin{aligned}
& \Delta m_{12}^{2}=8.2 \times 10^{-5} \mathrm{eV}^{2} \\
& \Delta m_{23}^{2}=2.4 \times 10^{-3} \mathrm{eV}^{2} .
\end{aligned}
$$

From Eqs. (25)-(29), we obtain the matrix $\mathbf{S}_{\nu}$ as a function of $M$, with which we numerically solve the RGEs and obtain the Higgs boson mass bounds as a function of $M$. The window for 
the Higgs boson pole mass for both the hierarchical and inverted-hierarchical cases is shown in Fig. 4. As $M$, or equivalently the Yukawa couplings become large, the window for the Higgs boson mass becomes narrower and is eventually closed.

As previously mentioned, for low $M$ values, the Higgs boson mass bounds with type III seesaw are different from the SM ones and the range of the Higgs boson mass window is enlarged. This result can be qualitatively understood in the following way. The presence of the triplet fermions significantly alters the RGE running of the $\mathrm{SU}(2)_{L}$ gauge coupling by making it asymptotically non-free, so that $g_{2}(\mu)$ for $\mu>M$ is larger than the SM value without type III seesaw. In the analysis of the vacuum stability bound, the Higgs quartic coupling is small so that Eq. (11) can be approximated as

$$
\beta_{\lambda}^{(1)} \simeq \frac{9}{4}\left(\frac{3}{25} g_{1}^{4}+\frac{2}{5} g_{1}^{2} g_{2}^{2}+g_{2}^{4}\right)-12 y_{t}^{4} .
$$

The first term on the right hand side is larger in type III seesaw than in the SM case and as a result, the Higgs quartic coupling decreases more slowly than in the SM. Consequently, the vacuum stability bound on the Higgs boson mass is lowered. For the perturbativity bound, the Higgs quartic coupling is large and Eq. (11) can be approximated by

$$
\beta_{\lambda}^{(1)} \simeq 12 \lambda^{2}-\left(\frac{9}{5} g_{1}^{2}+9 g_{2}^{2}\right) \lambda+12 y_{t}^{2} \lambda-12 y_{t}^{4},
$$

The beta function is smaller than the SM one due to the second term. Therefore, the evolution of the Higgs quartic coupling is slower, and as a result, the Higgs boson mass based on the perturbative bound is somewhat larger than the SM one.

Finally, it is interesting to compare the results in type III seesaw with those in type I seesaw [8]. We examine type I seesaw with three singlet fermions with a degenerate mass $M$. The RGE formulas for type I seesaw are slightly different from those in type III. The RGEs for the gauge couplings are the same as in the SM because only SM singlet fields are introduced. The RGEs corresponding to Eqs. (22) $-(24)$ are given by

$$
\begin{aligned}
& \beta_{t}^{(1)} \rightarrow \beta_{t}^{(1)}+\operatorname{tr}\left[\mathbf{S}_{\nu}\right], \\
& 16 \pi^{2} \frac{d \mathbf{S}_{\nu}}{d \ln \mu}=\mathbf{S}_{\nu}\left[6 y_{t}^{2}+2 \operatorname{tr}\left[\mathbf{S}_{\nu}\right]-\left(\frac{9}{10} g_{1}^{2}+\frac{9}{2} g_{2}^{2}\right)+3 \mathbf{S}_{\nu}\right], \\
& \beta_{\lambda}^{(1)} \rightarrow \beta_{\lambda}^{(1)}+4 \operatorname{tr}\left[\mathbf{S}_{\nu}\right] \lambda-4 \operatorname{tr}\left[\mathbf{S}_{\nu}{ }^{2}\right] .
\end{aligned}
$$

In type I seesaw, we define the light neutrino mass matrix as $\mathbf{M}_{\nu}=-\frac{v^{2}}{2 M} \mathbf{Y}_{\nu}^{T} \mathbf{Y}_{\nu}$, as usual.

We repeat the previous analysis with the RGEs for the type I seesaw and the results are shown in Fig. 5, to be compared with the results for type III shown in the Fig. 4. We find qualitatively the same behavior for the Higgs boson mass bounds for a large seesaw scale or 
equivalently large Yukawa couplings. An important difference between type I and III seesaws can be seen for a small seesaw scale or equivalently small Yukawa couplings. In type I seesaw, the Higgs boson mass bounds reduce to the SM results, because only the Yukawa couplings affect the RGEs in this case.

In conclusion, we have considered the potential impact of type III seesaw on the vacuum stability and perturbativity bounds on the Higgs boson mass. For energies higher than the seesaw scale, the triplet fermions introduced in type III seesaw are involved in quantum corrections and the RGEs of the SM are modified. There are two important effects. One is the neutrino Yukawa coupling contribution and the other is the modification of the RGE of the $\mathrm{SU}(2)_{L}$ gauge coupling due to the presence of the triplet fermions. We have found that as the Yukawa couplings are increased, the vacuum stability bound grows and eventually merges with the perturbativity bound. Therefore, the Higgs boson mass window is closed at some large Yukawa couplings with a fixed seesaw scale, or some high seesaw scale by fixing the light neutrino mass scale. Even if the new Yukawa couplings are negligible, there is a remarkable effect due to the modification of the RGE evolution of the $\mathrm{SU}(2)_{L}$ gauge coupling. For a low seesaw scale, the Higgs boson mass window between the vacuum stability and perturbative bounds turns out to be wider than the SM one. This is in contrast with type I seesaw where the Higgs boson mass bounds in the SM are reproduced in the small Yukawa coupling limit.

\section{Acknowledgments}

This work is supported in part by the DOE Grant \# DE-FG02-91ER40626 (I.G. and Q.S.), and the Grant-in-Aid for Scientific Research from the Ministry of Education, Science and Culture of Japan, \#18740170 (N.O.).

\section{References}

[1] I. Gogoladze, N. Okada and Q. Shafi, arXiv:0802.3257 [hep-ph].

[2] G. Lazarides, Q. Shafi and C. Wetterich, Nucl. Phys. B181, 287 (1981); R. N. Mohapatra and G. Senjanović, Phys. Rev. D 23, 165 (1981); M. Magg and C. Wetterich, Phys. Lett. B 94, 61 (1980); J. Schechter and J. W. F. Valle, Phys. Rev. D 22, 2227 (1980).

[3] N. Cabibbo, L. Maiani, G. Parisi and R. Petronzio, Nucl. Phys. B 158, 295 (1979); P. Q. Hung, Phys. Rev. Lett. 42, 873 (1979); M.A.B. Beg, C. Panagiotakopoulos and A. Sirlin, Phys. Rev. Lett.52 (1984) 883; K. S. Babu and E. Ma, Phys. Rev. Lett. 55, 3005 (1985); M. Lindner, Z. Phys. C 31, 295 (1986); M. Sher, Phys. Rept. 179, 273 (1989); 
G. Altarelli and G. Isidori, Phys. Lett. B 337, 141 (1994); J. A. Casas, J. R. Espinosa and M. Quiros, Phys. Lett. B 342, 171 (1995); Phys. Lett. B 382, 374 (1996); J. R. Espinosa and M. Quiros, Phys. Lett. B 353, 257 (1995).

[4] R. Barate et al. [LEP Working Group for Higgs boson searches], Phys. Lett. B 565, 61 (2003).

[5] P. Minkowski, Phys. Lett. B 67, 421 (1977); T. Yanagida, in Proceedings of the Workshop on the Unified Theory and the Baryon Number in the Universe (O. Sawada and A. Sugamoto, eds.), KEK, Tsukuba, Japan, 1979, p. 95; M. Gell-Mann, P. Ramond, and R. Slansky, Supergravity (P. van Nieuwenhuizen et al. eds.), North Holland, Amsterdam, 1979, p. 315; S. L. Glashow, The future of elementary particle physics, in Proceedings of the 1979 Cargèse Summer Institute on Quarks and Leptons (M. Lévy et al. eds.), Plenum Press, New York, 1980, p. 687; R. N. Mohapatra and G. Senjanović, Phys. Rev. Lett. 44, 912 (1980).

[6] B. T. Cleveland et.al, Astrophys.J. 496505 (1998); Super-Kamiokande Collaboration, Phys. Lett. B539 179 (2002); Super-Kamiokande Collaboration, Phys. Rev. D71 112005 (2005); M. Maltoni, T. Schwetz, M.A. Tortola, J.W.F. Valle New J.Phys. 6122 (2004); A. Bandyopadhyay et al, Phys. Lett. B608 115 (2005); G. L. Fogli et al, Prog. Part. Nucl. Phys. 57742 (2006); For a recent review, see, for example, H. Nunokawa, S. J. Parke and J. W. F. Valle, Prog. Part. Nucl. Phys. 60, 338 (2008).

[7] R. Foot, H. Lew, X. G. He and G. C. Joshi, Z. Phys. C 44, 441 (1989).

[8] J. A. Casas, V. Di Clemente, A. Ibarra and M. Quiros, Phys. Rev. D 62, 053005 (2000).

[9] [CDF Collaboration], arXiv:0803.1683 [hep-ex].

[10] W. M. Yao et al. [Particle Data Group], J. Phys. G 33 (2006) 1.

[11] M. E. Machacek and M. T. Vaughn, Nucl. Phys. B 222, 83 (1983); Nucl. Phys. B 236, 221 (1984); Nucl. Phys. B 249, 70 (1985); C. Ford, I. Jack and D. R. T. Jones, Nucl. Phys. B387 (1992) 373, [Erratum-ibid. B504 (1997) 551]; H. Arason, D. J. Castano, B. Keszthelyi, S. Mikaelian, E. J. Piard, P. Ramond and B. D. Wright, Phys. Rev. D 46, 3945 (1992); V. D. Barger, M. S. Berger and P. Ohmann, Phys. Rev. D 47, 1093 (1993); M. X. Luo and Y. Xiao, Phys. Rev. Lett. 90, 011601 (2003). 
[12] See, for example, H. Arason, D. J. Castano, B. Keszthelyi, S. Mikaelian, E. J. Piard, P. Ramond and B. D. Wright, Phys. Rev. D 46, 3945 (1992); H. E. Haber, R. Hempfling and A. H. Hoang, Z. Phys. C 75, 539 (1997).

[13] See, for example, F. Jegerlehner, M. Y. Kalmykov and O. Veretin, Nucl. Phys. B 641, 285 (2002); Nucl. Phys. B 658, 49 (2003); F. Jegerlehner and M. Y. Kalmykov, Nucl. Phys. B 676, 365 (2004).

[14] A. Sirlin and R. Zucchini, Nucl. Phys. B 266 (1986) 389.

[15] P. F. Harrison, D. H. Perkins, W. G. Scott, Phys. Lett. B530 167 (2002). 


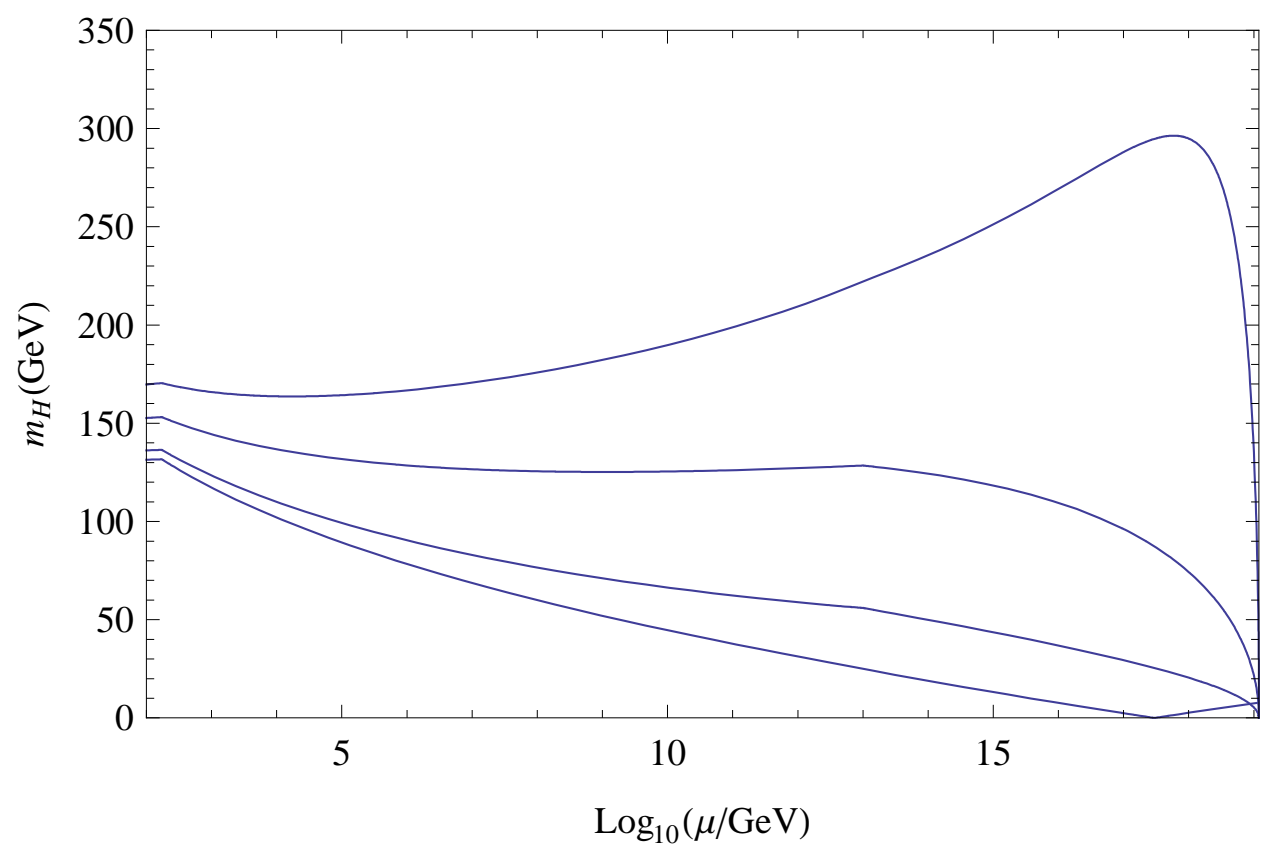

Figure 1: Evolution of running Higgs mass $\left(m_{H}(\mu)=\sqrt{\lambda(\mu)} v\right)$ corresponding to the vacuum stability bound for various $Y_{\nu}$ values and the seesaw scale $M=10^{13} \mathrm{GeV}$. Each line corresponds to $Y_{\nu}=1.5,1.2,0.8$ and 0 from top to bottom.

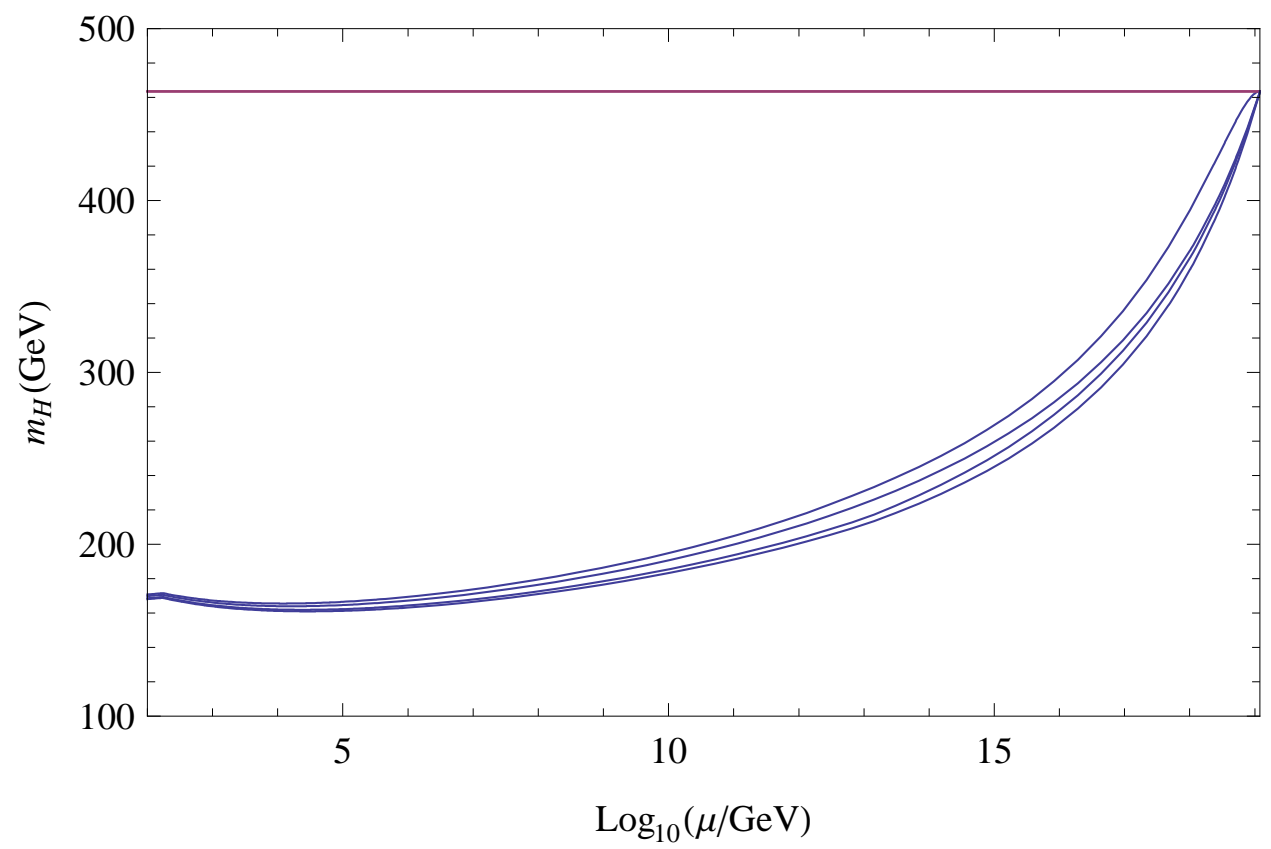

Figure 2: Evolution of running Higgs mass $\left(m_{H}(\mu)=\sqrt{\lambda(\mu)} v\right)$ corresponding to the perturbativity bound for various $Y_{\nu}$ values and the seesaw scale $M=10^{13} \mathrm{GeV}$. Each line corresponds to $Y_{\nu}=1.5,1.2,0.8$ and 0 from top to bottom. The horizontal line corresponds to $m_{H}\left(M_{P l}\right)=(4 \pi)^{1 / 4} v=464 \mathrm{GeV}$. 


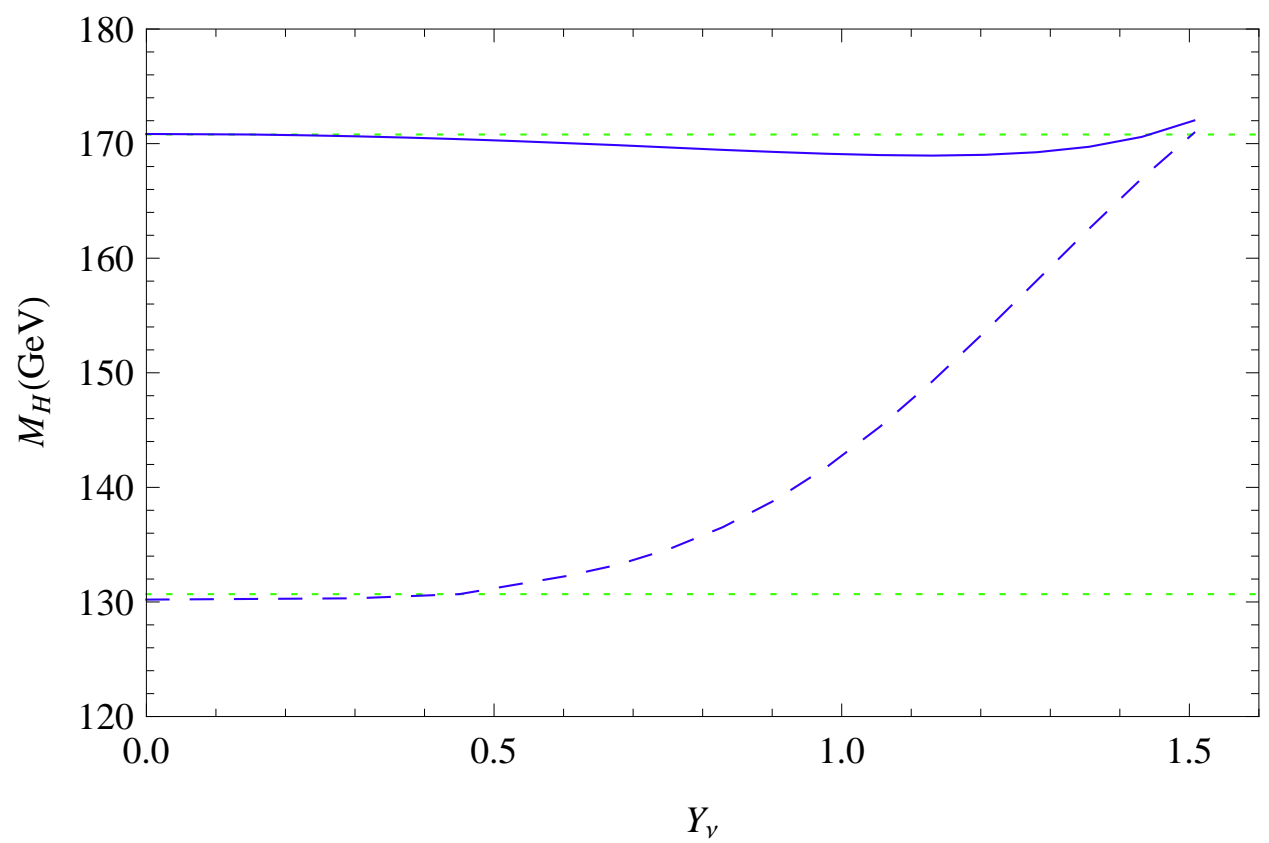

Figure 3: Perturbativity (solid) and vacuum stability (dashed) bounds on the Higgs boson pole mass $\left(M_{H}\right)$ versus $Y_{\nu}$ with the seesaw scale $M=10^{13} \mathrm{GeV}$. The upper and lower dotted lines respectively show the perturbativity bound $\left(M_{H} \simeq 171 \mathrm{GeV}\right)$ and the vacuum stability bound $\left(M_{H} \simeq 131 \mathrm{GeV}\right)$ in the SM case.

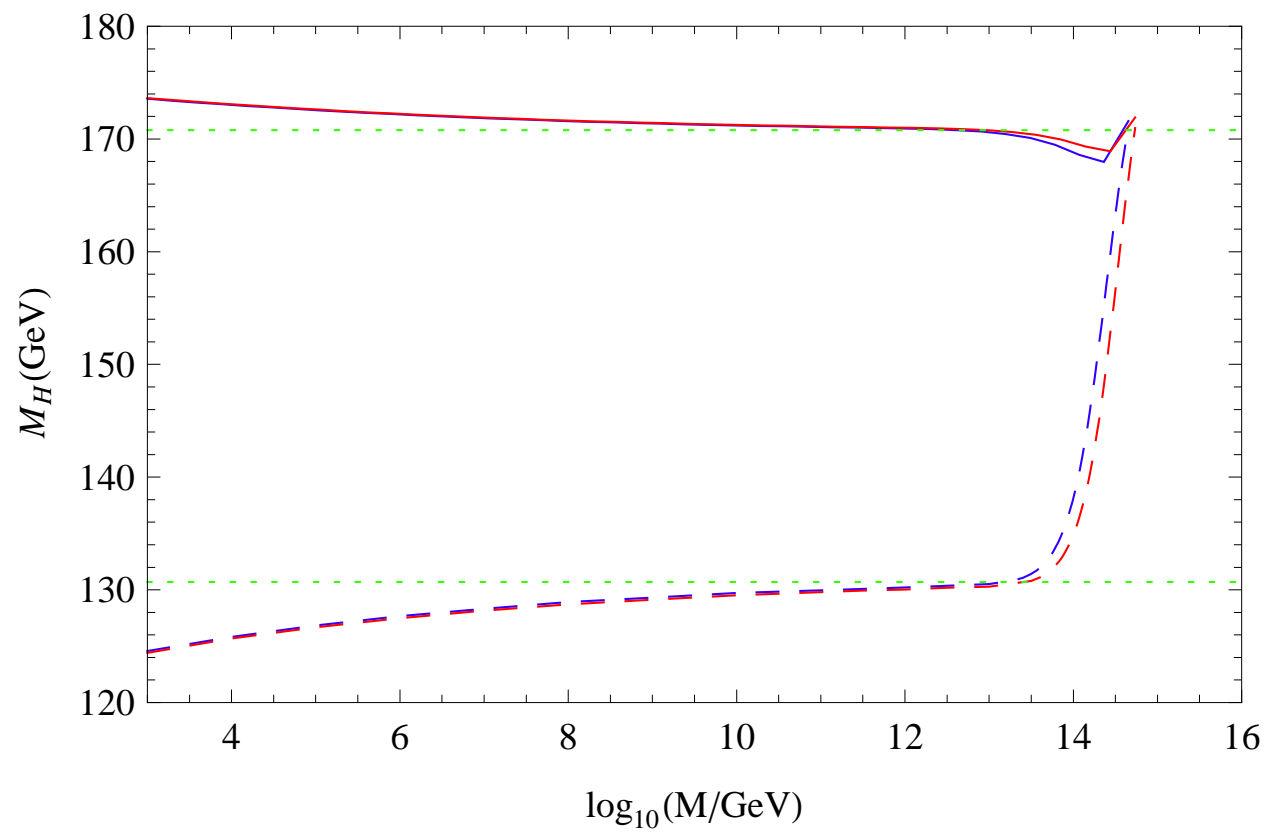

Figure 4: Perturbativity and vacuum stability bounds versus $M$, with a hierarchical mass spectrum (outer region in red), and an inverted-hierarchical mass spectrum (inner region in blue). 


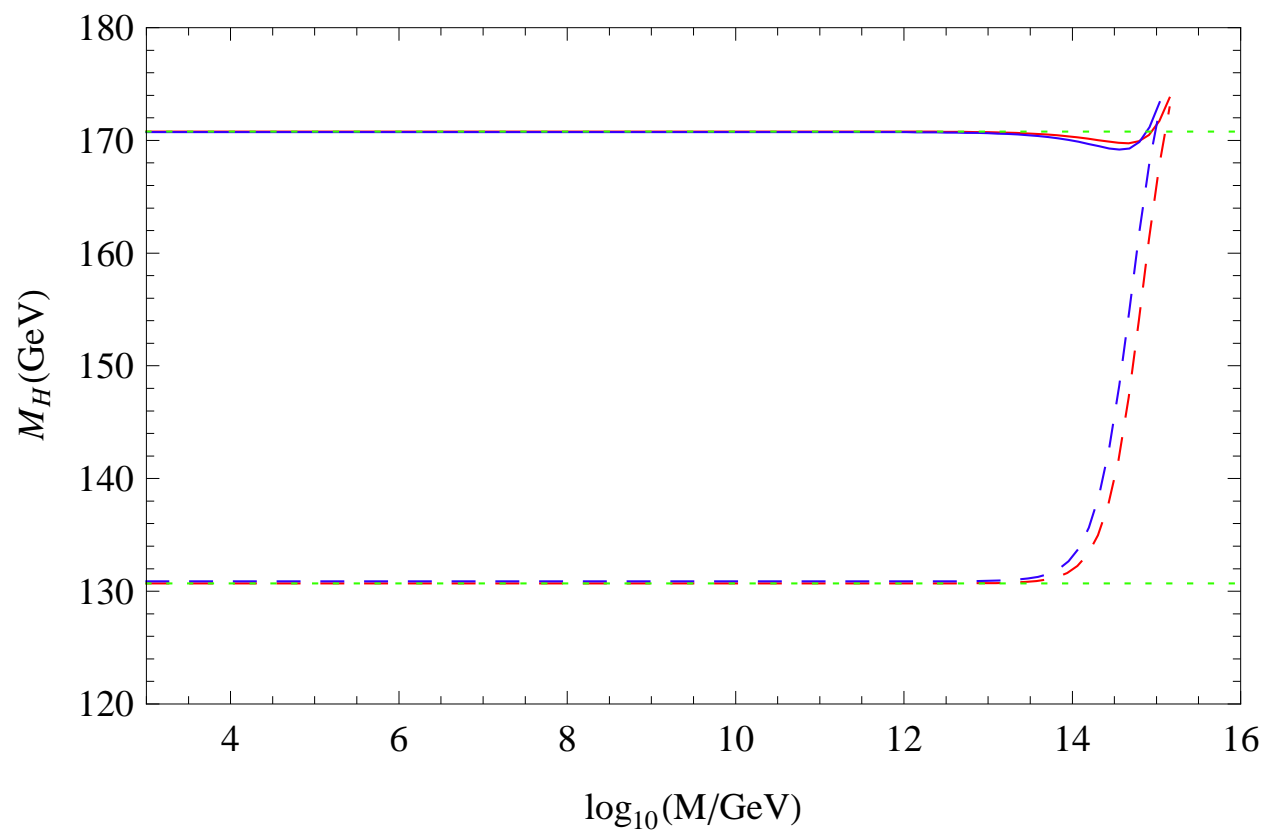

Figure 5: Similar to Figure 4, but for type I seesaw with three singlet neutrinos. 